\title{
The Physical Aspect of Action of Biologically Active Substances in Ultra- Low Doses and Low-Intensity Physical Factors on Biological Objects: Spin Supercurrents
}

\section{Boldyreva LB*}

State University of Management, Moscow, Russia

\begin{abstract}
The paper aims at showing that there is a physical process that has the same features as the effects of ultra-low doses of biologically active substances and low-intensity physical factors, such as ionizing radiation and non-ionizing electromagnetic radiation, on biological objects.

An analysis is given of the main features of the above effects, namely: the non-monotonic dose-effect dependence, the kinetic paradox, the change in sensitivity of the biological object with respect to a subsequent exposure to ultra-low doses, the dependence of the 'sign' of the effect on the initial state of the target biological object.
\end{abstract}

Keywords: Alternative medicine; Ultra-low doses; Ultra-low doses of biologically active substances; Low-intensity ionizing radiation; Lowintensity non-ionizing electromagnetic radiation; Spin supercurrent; Spin structures in physical vacuum; Model of superfluid physical vacuum

Abbreviations: BO: Biological Object; ULD: Ultra-Low Dose; BAS: Biologically Active Substance; HPD: Homogeneously Precessing Domain

\section{Introduction}

The paper concerns the mechanism of action on biological objects (BO) of the following substances/physical factors in ultra-low doses (ULD): biologically active substances (BAS), concentrations of $10^{-13} \mathrm{M}$ or lower [1]; ionizing radiation, the one-time equivalent dose of radiation is less than $0.1 \mathrm{~Sv}$ [2]; non-ionizing electromagnetic radiation, the energy flux density is less than $1 \mu \mathrm{W} / \mathrm{cm}^{2}$ [1]. The levels of biological organization at which the action of ULDs are revealed are quite various: from macromolecules, cells, organs, tissues to plants and animals. The studies [1,3-5] have shown that the effects of BAS in ULD and the effects of low-intensity ionizing and non-ionizing radiation on biological objects have a number of similar features. Some of them are discussed below:

1. The non-monotonic, polymodal dose-effect dependence. In most cases the activity maxima are observed within definite ranges of doses, which are separated by so-called "dead zones". In some cases, the same effects are produced by low-doses differing in 5 to 8 orders of magnitude. There are also cases where a change in the "sign" of the effect is observed in the dose dependence. Figures 1-4 give some examples of this feature.

Figure 1 shows the type of variation of the content of protein p53 with mice of $\mathrm{F} 1$ line as a function of dose $D$ of the injected BAS, namely, antioxidant phenosan [6,7]. The value $D=10^{-14} \mathrm{~mole} / \mathrm{kg}$ corresponds to an ultra-low dose.

Figure 2 shows the type of dependence of human mortality (caused by leukemia) on the equivalent dose $d$. As the death rate $K$ the ratio of the number of deaths per 100000 person-years to the number of deaths caused by the equivalent dose of about $23 \mathrm{mSv}$ is used. The curve is based on the data collected under Burlakova's guidance $[2,8]$. It is noteworthy that there is a range of values of $d$ (at about $75 \mathrm{mSv}$ ) where the magnitude of $K$ is less than that for the background value of $d$ (about $2 \mathrm{mSv}$ ). It may be said that ultra-low doses of ionizing radiation in this range has a therapeutic effect.

Figures 3-5 show some results of the experiment where test group

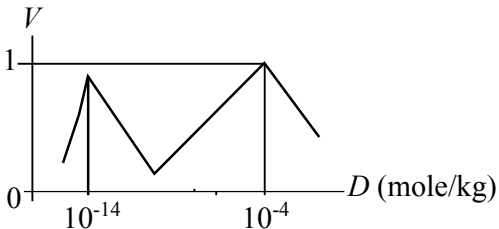

Figure 1: The type of dependence of the normalized (to the maximum value, at $D=10^{-4} \mathrm{~mole} / \mathrm{kg}$ ) value $V$ of content of protein p53 with mice of $\mathrm{F} 1$ line as a function of dose $D$ of the injected antioxidant phenosan.

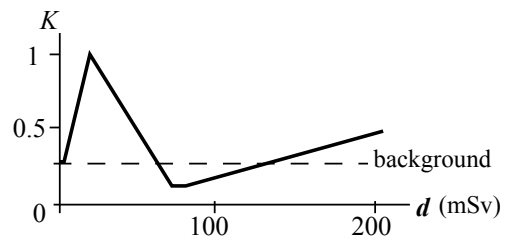

Figure 2: The type of dependence of human mortality (caused by leukemia) on the equivalent dose $d . K$ is the ratio of the number of deaths per 100000 person-years caused by arbitrary value of equivalent dose $d$ to the number of deaths caused by the equivalent dose of about $23 \mathrm{mSv}$.

*Corresponding author: Liudmila B Boldyreva, PhD, Associate Professor, the State University of Management, Moscow, Russia, E-mail: boldyrev-m@yandex.ru Received March 21, 2013; Accepted April 12, 2013; Published April 15, 2013

Citation: Boldyreva LB (2013) The Physical Aspect of Action of Biologically Active Substances in Ultra-Low Doses and Low-Intensity Physical Factors on Biological Objects: Spin Supercurrents. Altern Integ Med 2: 110. doi:10.4172/23275162.1000110

Copyright: (c) 2013 Boldyreva LB. This is an open-access article distributed under the terms of the Creative Commons Attribution License, which permits unrestricted use, distribution, and reproduction in any medium, provided the original author and source are credited. 




Figure 3: The difference $Y$ between the blood clotting time for test group rats and that for control group rats (not exposed to the magnetic field), divided by the blood clotting time for control group, against the magnetic field strength $H$. The frequency $f$ of the magnetic field is $5 \mathrm{~Hz}$.

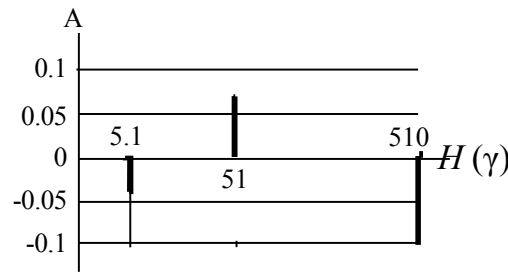

Figure 4: The difference $A$ between the erythrocyte count in the blood of test group rats and that in the blood of control group rats (not exposed to the magnetic field), divided by the erythrocyte count in the blood of control group, against the magnetic field strength $H$. The frequency $f$ of the magnetic field is $10 \mathrm{~Hz}$.

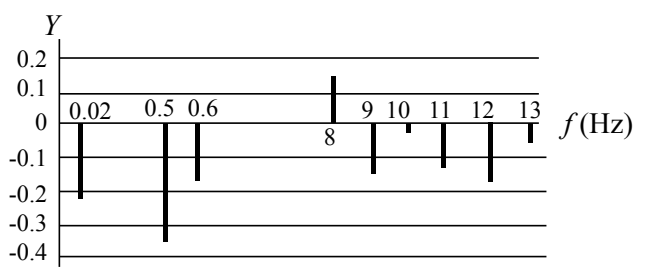

Figure 5: The difference $Y$ between the blood clotting time for test group rats and that for control group rats (not exposed to the magnetic field), divided by the blood clotting time for control group, against the magnetic field frequency $f$. The magnetic field strength is $51 \gamma$.

rats were exposed to low-frequency alternating magnetic field. Figure 3 shows the difference $Y$ between the normalized blood clotting time for test group rats (exposed to the magnetic field) and that for control group rats (not exposed to the magnetic field) against the magnetic field strength $H$. The frequency $f$ of the magnetic field is $5 \mathrm{~Hz}$. Helmholtz coils were used to produce the magnetic field. Figure 4 shows the difference A between the normalized erythrocyte count in the blood of test group rats and that of control group rats (not exposed to the magnetic field) against the magnetic field strength $H$. The frequency $f$ of the magnetic field is $10 \mathrm{~Hz}$ [9].

2. The kinetic paradox. As concerns the effects of BAS in ULD on $\mathrm{BO}$ such as a cell or an organism, the kinetic paradox means that the effect is the strongest when the $\mathrm{BO}$ already contains the same substance but in a dose that is some orders of magnitude greater than the ULD used. In the case of effects of non-ionizing electromagnetic radiation on $\mathrm{BO}$ this means, according to the model discussed below, that there are frequency ranges, or even single frequencies, where the effect is revealed. A change in the "sign" of the effect can take place. An example of this is shown in Figure 5. In the experiment the test group rats were exposed to low-frequency alternating magnetic field. The Figure shows the difference $Y$ between the normalized blood clotting time for test group rats and that for control group rats (not exposed to the magnetic field) as a function of magnetic field frequency $f$. The magnetic field strength is equal to $51 \gamma$ [9]

3. The change in sensitivity of $\mathrm{BO}$ with respect to a subsequent exposure to ULD. Among the examples that support this feature are the following.

-The equivalent dose of ionizing radiation received during a short period of time causes less radiation injury than the same equivalent dose received during much longer period of time [10].

-If in the course of a long-continued low-dose irradiation an additional short irradiation occurs, this produces a more pronounced effect than that resulting from a single exposure to the total dose of those irradiations [2].

-The efficacy of remedies in ultra-low (homeopathic) doses is the greatest when they are administered repeatedly.

4. The dependence of the "sign" of the effect (inhibition or stimulation) on the initial state of the $\mathrm{BO}$ being treated. This is observed in almost every type of effect of ULD on BO.

Extensive research is conducted of synergetic effects of ultralow doses of medicines and other agents. It has been shown also that exposure of a biological object to BAS in ULD or low-intensity physical factors increases the sensitivity of the $\mathrm{BO}$ to subsequent exposure of the latter to other (or the same) agents in high doses. For example, radiation therapy of malignant tumors (those of intestines, lung, or breast) appears to be more efficient if the tumor is first irradiated with the dose of 10cGy or lower and then with a therapeutic dose of 1,9Gy than in the case where there is no preliminary irradiation with low doses [11].

The studies of joint action of a few medicines, one of which being administered in ULD, proved to be very promising. For example, the toxin ricin in ULD of $10^{-15}-10^{-18} \mathrm{M}$ enhances the synthesis of cytokines by lymphoid cells, which results in death of tumor cells [12].

The results of numerous studies of the effects of radiation and other physical and chemical factors can be found in the UNSCEAR reports $[5,13]$.

The study of the effects of the above factors on BO has shown that it is extremely difficult to explain the effects on the basis of modern physics. The concepts and principles of statistical physics do not apply to the effects of ultra-low doses on biological objects. To the author's knowledge, in what concerns the effects of BAS and physical factors (in particular, ionizing and non-ionizing radiation) on $\mathrm{BO}$, the changeover from large doses to ultra-low doses means, theoretically, a changeover from the statistical approach to the description of the effects to the deterministic approach.

According to quantum field theory, quantum entities create pairs of virtual particles, or particle-antiparticle pairs, in the physical vacuum. For the virtual particles the classical relation between mass, energy and momentum does not hold; however, they have spin which is the same as for the real particles. Hence it follows that 1) spin has no definite direction, and by the magnitude of spin the magnitude of its projection onto a preferential direction is meant; this can be interpreted as a precession of the spin about the preferential direction and allows one to introduce the frequency of the precession, the angles of precession and nutation;

2) spin correlations can take place. Thus all bodies as consisting of quantum entities may produce in the physical vacuum the spin structures consisting of virtual particles, the structures being characterized by 
respective frequencies of precession, precession angles and nutation angles, and spin correlations can exist between such structures.

All types of ULD considered in this paper consist of quantum entities. In the case of BAS these are atoms and molecules of the substances which are introduced into the $\mathrm{BO}$; in the case of nonionizing electromagnetic radiation these are photons of radiation; and in the case of ionizing radiation these are "secondary" quantum entities produced in the BO during exposure to the ionizing radiation. "Secondary" quantum entities may be ions, neutrons, free electrons, and other particles. Besides, if the ionizing radiation is a gammaradiation ( $\Upsilon$-rays), the Compton effect can take place: the scattering of high energy photons by free electrons, producing recoil electrons and scattered photons. From now on, for brevity sake the quantum entities relating to all types of ULD, which are discussed in this paper, will be referred to as "quantum entities that constitute the ULD".

It will be shown in this paper that all of the above features 1-4 of the effects of ULD on BO can be described by the spin correlations between the spin structures produced in the physical vacuum by "quantum entities that constitute ULD" and the spin structures produced in the physical vacuum by quantum entities that constitute the $\mathrm{BO}$ provided the properties of the spin correlations are like the properties of spin supercurrents in superfluid ${ }^{3} \mathrm{He}-\mathrm{B}$.

Note. The above mentioned spin correlations can account for the influence of cavity structures on biological objects. There is much evidence of cavity structure influencing BO [14-16]. In Europe, Oskar Korschelt was likely the first who was granted a patent for the use of specially fabricated cavity structures for medical purposes [17]. He made alternating cavities out of metal (Figure 6a) and used them to treat stomach problems, nerve diseases, insomnia and pains. In Russia the medical aspect of cavity structures was studied by Grebennikov $[16,18]$, and there is a museum in Novosibirsk where a device containing bee combs (Figure 6b) intended for treating patients is exhibited. The studies have shown that bee combs can influence respiratory organs, organs of hearing, give rise to feeling of loss of weight of arms, legs or body as a whole. The cavity structure in its effects on $\mathrm{BO}$ can be seen as a low-intensity physical factor.

\section{Methods and Results}

The properties of spin supercurrents in superfluid ${ }^{3} \mathrm{He}-\mathrm{B}$ and features of effects of ultra-low doses of biologically active substances and low-intensity physical factors on biological objects

In superfluid ${ }^{3} \mathrm{He}-\mathrm{B}$ there may exist spin structures where coherent precession of spins of ${ }^{3} \mathrm{He}$ atoms takes place. Such a structure is called a homogeneously precessing domain (HPD) [19-22]. An HPD is characterized by spin $S$, precession angle (phase) $\alpha$, nutation angle $\beta$, and precession frequency $\omega$ (Figure 7).

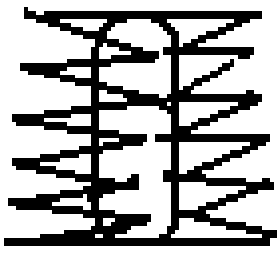

(a)

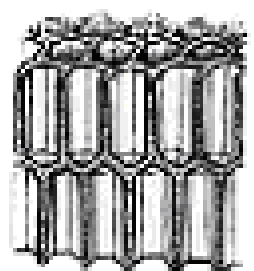

(b)
Figure 6: Examples of cavity structures: (a) made by $\mathrm{O}$. Korschelt out of metal; (b) bee combs.

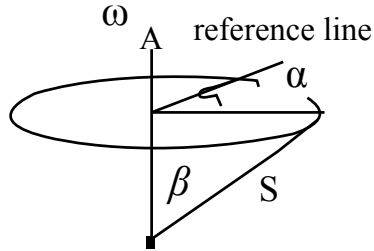

Figure 7: The diagram of the precession of spin $S$ with frequency $\omega ; \alpha$ is the precession angle relative to a reference line, $\beta$ is the nutation angle.
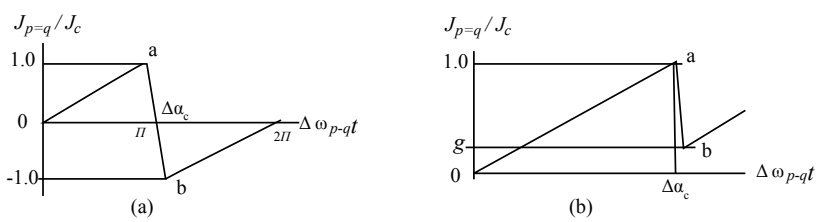

(b)

Figure 8: Normalized spin supercurrent $J_{p-q} / J_{c}$ as a function of $\Delta \omega_{p-q} t$ ( $t$ is time, $\Delta \omega_{p-q}$ is the difference in precession frequencies of $H P D$ and $H P D_{q}$ ). The line $a-b$ corresponds to the phase slippage by the value of $2 \pi$. For (a) $\Delta \alpha_{c}=\pi$, for (b) $\Delta \alpha_{c}>2 \pi ; g=1-b_{1} 2 \pi$.

In a homogeneously precessing domain, energy $\mathrm{U}$ is related to the frequency $\omega$ of precession as:

$U=S \omega$.

The precession and nutation angles are order parameters for superfluid ${ }^{3} \mathrm{He}-\mathrm{B}$, and there are processes that tend to make equal the respective order parameters throughout the whole volume of the superfluid. Such processes in superfluid ${ }^{3} \mathrm{He}-\mathrm{B}$ are spin supercurrents. In the case where the precession frequencies are directed along axis $z$, the spin supercurrent component in the direction of axis $z, J_{z}$, is determined as:

$$
J_{z}=-b_{1} \frac{\partial \alpha}{\partial z}-b_{2} \frac{\partial \beta}{\partial z},
$$

where $b_{1}$ and $b_{2}$ are proportionality factors dependent on $\beta$ and the properties of the medium. Using the expression

$$
\alpha=\omega t+\alpha_{0},
$$

where $\alpha_{0}$ is the value of precession angle at $t=0$, the spin supercurrent $J_{p-q}$ between two arbitrary HPD (HPD and $\left.H P D_{q}\right)$ may be expressed by the difference in their precession frequencies $\Delta \omega_{p-q}$ and time $t$. In the special case where the nutation angles of those HPD are equal and their precession frequencies are aligned and do not depend on time, the expression for $J_{p-q}$ can be written as:

$$
J_{p-q}=b_{1} \Delta \omega_{p-q} t .
$$

We assume that the value of the total spin $S$ is taken to be constant for the interacting homogeneously precessing domains and $J_{p-q}=0$ at $t=0$. According to (2) and (3), the spin supercurrent is directed from the HPD having a greater precession frequency to the HPD having a smaller precession frequency.

There exists such a phenomenon in ${ }^{3} \mathrm{He}-\mathrm{B}$ as phase slippage. At a definite difference in precession angles, $\Delta \alpha_{c}$, determined by the properties of the superfluid medium, a precession phase slippage (drop) by the value of $2 \pi n$ takes place. The critical spin supercurrent $J_{c}$ corresponds to the value $\Delta \alpha_{c}$. Figure 8 shows examples of experimental dependences of the normalized spin supercurrent $J_{p-q} / J_{c}$ between $H P D_{p}$ and $H P D_{q}$ on the value of $\Delta \omega_{p-q} t$ provided this value may exceed $\Delta \alpha_{c}$ [19-22]. On the curves of Figure 8, the line 
Citation: Boldyreva LB (2013) The Physical Aspect of Action of Biologically Active Substances in Ultra-Low Doses and Low-Intensity Physical Factors on Biological Objects: Spin Supercurrents. Altern Integ Med 2: 110. doi:10.4172/2327-5162.1000110

$a-b$ corresponds to the change in the supercurrent in the process of phase slippage by the value of $2 \pi ; \Delta \omega_{p-q}$ does not depend on time. In Figure 8a the phase slip is shown to take place at $\Delta \alpha_{c}=\pi$ and the spin supercurrent changes its sign. On the curves of Figure $8 \mathrm{~b}$ the phase slip occurs at $\Delta \alpha_{c}>2 \pi$, the spin supercurrent not changing its sign. In this case the value $J_{p-q} / J_{c}$ after the phase slip (this value is denoted by g) is determined as $g=1-b_{1} 2 \pi$.

If spin correlations between the spin structures produced in the physical vacuum by "quantum entities that constitute the ULD" and the spin structures produced in the physical vacuum by quantum entities that constitute the $B O$ are like spin supercurrents in superfluid $3 H e-B$, then the first feature of the effects of ULD on BO (see Introduction), i.e. the non-monotonic, polymodal dose-effect dependence, is analogous to the phase slip effect.

Generally, the determination of time dependency of the magnitude of the spin supercurrent between two HPD is a difficult problem, because the speed of transmission of information of the existence of order parameter gradient is, in theory, infinite, and the speed of the spin supercurrent is finite. Besides, a possibility of phase slippage should be taken into account. The respective precession and nutation angles of the interacting HPDs will become equal, provided the distance $X$ between them and the difference between their precession frequencies, $\Delta \omega$, satisfy the following conditions:

$$
X \rightarrow 0 \text {, }
$$

$\Delta \omega \rightarrow 0$.

These two conditions are analogous to the second feature of the effects of ULD on BO, see Introduction, i.e. the kinetic paradox. Condition (6) is always valid for those $B A S$ which are contained in the BO but in doses some orders of magnitude higher than the ULD used. The high concentration of such a substance in the BO is necessary for the spin structure produced by the $B O$ in the physical vacuum to have the characteristics determined by the properties of the substance.

The similarity in the structures of expressions determining the photon energy and the HPD energy (1) allows us to suppose that the frequency of a photon is equal to the frequency of precession of spins in the spin structure produced by the photon in the physical vacuum. In this case the non-ionizing electromagnetic radiation will exert action on $B O$ if the frequency of photon is of the same order of magnitude as the precession frequency of the spin structure produced by $B O$, that is, the condition (6) is valid for these frequencies.

Since spin supercurrents tend to make the respective characteristics of interacting spin structures equal, then, providing such equalization is possible, the value $\Delta \omega_{p-q}$ for arbitrary spin structures $H P D_{p}$ and $H P D_{q}$ will decrease. That is, the following is valid:

$$
\left|\Delta \omega_{p-q}\right|_{\tau_{2}}<\left|\Delta \omega_{p-q}\right|_{\tau_{1}}
$$

where $\left|\Delta \omega_{p-q}\right|_{\tau_{1}}$ and $\left|\Delta \omega_{p-q}\right|_{\tau_{2}}$ are differences between precession frequencies of $H P D_{p}$ and $H P D_{q}$ respectively at time $\tau_{1}$ and at time $\tau_{2}, \tau_{2}>\tau_{1}$.

Let us correlate this conclusion with the third feature of the effects of $U L D$ on $B O$, see Introduction, that is, the change in sensitivity of the $B O$ with respect to a subsequent exposure to ULD. The action of ULD on a $B O$ affects the characteristics of the spin structure produced by the $B O$ in the physical vacuum. Consequently, in accordance with (7), after the first exposure to ULD the condition (6) may be valid for a subsequent exposure of the BO to ULD, although before the first exposure to ULD the condition (6) was not valid. And vice versa: condition (6) may appear not to be valid for subsequent exposure of the BO to ULD, although before the first exposure to ULD the condition (6) was valid. (Condition (5) is taken to be always valid).

According to (1), changes in $\omega$ results in changes in the HPD energy. Thus as a result of action of spin supercurrent, which changes the HPD frequency, the HPD energy may either increase or decrease depending on the sign of the change. Under (2)-(4) and (7), the direction of energy flow is the same as that of the spin supercurrent. Generally, according to (3), the direction of the spin supercurrent and, consequently, the energy flow direction depend on the characteristics (in particular $\alpha_{0}$ ) of the spin structure at the initial moment of time.

This property of spin supercurrent is analogous to the fourth feature of the effects of ULD on BO (see Introduction): the dependence of the "sign" of the effect on the initial state of the BO, if we associate the "sign" of the effect of ULD on the BO with the direction of energy flow between the respective spin structures produced by them in the physical vacuum.

The mutual position of HPDs in space may affect the characteristics of spin supercurrents between the HPDs. Let us consider two versions of configuration of a sequence of HPDs $\left(H P D_{1}, \ldots, H P D_{p}, \ldots, H P D_{q}\right.$, $\ldots, H P D_{r}$ ) having respective precession frequencies $\omega_{1}, \ldots, \omega_{p}, \ldots, \omega_{q}, \ldots$, $\omega_{r}$. In the first version all precession frequencies are aligned with axis $\mathbf{Z}$ (Figure 9a). If the respective characteristics of the above HPDs may be made equal, then for arbitrary $H P D_{p}$ and $H P D_{q} \Delta \omega_{p-q}=0$. In this case equation (4) is valid, and under this equation the spin supercurrent $J_{p-q}$ between arbitrary $H P D_{p}$ and $H P D_{q}$ may become zero, i.e. $J_{p-q}=0$. In the second version the sequence of HPDs makes up a ring, thus the straight line coincident with the axis $Z$ will become a circumference, see Figure $9 b$. If the ring of UPDs is a vortex ring in supercurrent ${ }^{3} \mathrm{He}-\mathrm{B}$, then as a result of the Barnett effect in superfluid ${ }^{3} \mathrm{He}-\mathrm{B}$ [23] the precession frequencies of the UPDs in question, $\omega_{1}, \ldots, \omega_{p}, \ldots, \boldsymbol{\omega}_{q}, \ldots, \boldsymbol{\omega}_{r}$, are tangential to the circumference, that is, the precession frequencies are not aligned with the same axis. In the latter case the spin supercurrent between any two HPDs will never be zero, and for spin supercurrent $J_{p-q}$ between arbitrary $H P D_{p}$ and $H P D_{q}$ the following holds:

$$
J_{p-q} \neq 0 .
$$

Thus the space between the HPDs that form a ring will be "filled" with spin supercurrents and consequently with the energy associated with the latter. (The non-zero spin supercurrents will be present even if a curved chain of HPDs is open, that is, does not make a ring.)

This property of spin supercurrents may determine the energy properties of cavity structures.

Now we shall consider the case where one HPD $\left(H P D_{0}\right)$ interacts with several HPDs $\left(H P D_{1}, \ldots, H P D_{w}\right)$. If the precession frequencies of

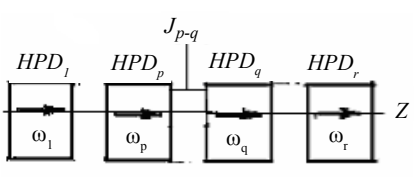

(a)

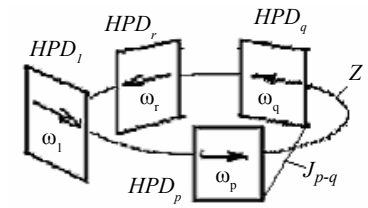

(b)
Figure 9: Two versions of sequence of HPDs $\left(H P D_{1}, \ldots, H P D_{p}, \ldots, H P D_{q}\right.$, $\ldots, H P D_{\text {f }}$ ), with respective precession frequencies $\omega_{1}, \ldots, \omega_{p}, \ldots, \omega_{q}, \ldots$, $\omega_{r}$, aligned with the tangent to Z: (a) the linear configuration, (b) the circle configuration. $J_{p-q}$ is spin supercurrent. 
with several HPDs $\left(H P D_{1}, \ldots, H P D_{w}\right)$. If the precession frequencies of all HPDs are aligned with the same axis, then according to (2) and (4) the total spin supercurrent $J_{\text {sum }}$ is defined as $j_{\text {sum }}=\sum_{i=1}^{w} j_{i}$, where $j_{i}$ is spin supercurrent between $H P D_{0}$ and $H P D_{i}$. Using (2) we obtain:

$$
j_{\text {sum }}=\sum_{i=1}^{w}\left(b_{1} \Delta \alpha_{i}+b_{2} \Delta \beta_{i}\right)
$$

where $\Delta \alpha_{i}$ is the difference in precession angles for $H P D_{0}$ and $H P D_{i}$, $\Delta \beta_{i}$ is the difference in nutation angles for $H P D_{0}$ and $H P D_{i}$. If all the values and signs of $\Delta \alpha_{i}$ and $\Delta \beta_{i}$ are respectively equiprobable and $w \rightarrow \infty$ then according to (9)

$$
j_{\text {sum }} \rightarrow 0 .
$$

In terms of the model of effects of biologically active substances and low-intensity physical factors (ionizing radiation and non-ionizing electromagnetic radiation) discussed in the present paper the condition (10) means that spin supercurrents cease to be the predominating factor that governs the effects and the effects will be determined by other physical factors.

\section{Discussion}

Since, according to the model in question, the action of biologically active substances in ultra-low doses and that of low-intensity physical factors, such as low-intensity ionizing radiation and non-ionizing electromagnetic radiation, on biological objects are determined by the same physical processes, they can produce the same effects on biological objects. There is much evidence on the relatively greater efficacy of combined action of these factors on biological objects. For example, small amounts of pesticides can increase the effect of low-dose ionizing radiation $[1,2]$. The same occurs for the effect of radiation in the presence of small amounts of mercury $[2,24]$

The interaction of quantum entities through spin supercurrents arise in a 'finer' physical medium (the physical vacuum) than the molecular one. Therefore, spin supercurrents cannot be shielded by molecular substances. This property of spin supercurrents agrees with the evidence given in the book by P. Bellavite and A. Signorine "The Emerging Science of Homeopathy" [25]: "There is some preliminary evidence demonstrating a homeopathic effect not only of solutions but also of closed ampoules containing solutions and placed in contact with the system to be regulated (human or animal)". This property of spin supercurrents may account for a paradoxical situation: a low-intensity electromagnetic radiation would exert action on a biological object through electromagnetic screens. The fact that cavity structures exert their influence on BOs independent of the presence of any screens between them, such as brick walls, metal shields, etc., can also be accounted for by the same property [16].

\section{Conclusion}

1. It is shown in the paper that the effects of ultra-low doses of biologically active substances and low-intensity physical factors (ionizing radiation and non-ionizing electromagnetic radiation) on biological objects have the features similar to those of the interaction between spin structures in superfluid ${ }^{3} \mathrm{He}-\mathrm{B}$ through spin supercurrents.

2. To low-intensity physical factors affecting biological objects belong as well the cavity structures, and these effects are caused by spin supercurrents which "fill" the cavity (and, therefore, by the energy associated with the supercurrents), the spin supercurrents being similar in their properties to spin supercurrents in the superfluid ${ }^{3} \mathrm{He}-\mathrm{B}$.
3. Although the model of action of biologically active substances in ultra-low doses and action of low-intensity physical factors discussed here is based on the principles of quantum mechanics (virtual pairs, spin supercurrents), it views the interaction between the quantum entities that constitute the substance/physical factor and the target biological object not as an interaction of ensembles (collections) of quantum entities but as an interaction of individual quantum entities. That is, not a statistical but a deterministic description of the interaction is used, which proceeds according to the laws governing the behavior of molecular liquid when the temperature of the latter is close to absolute zero (the properties of superfluid ${ }^{3} \mathrm{He}-\mathrm{B}$ ). This approach agrees with E. Schrödinger's point of view expressed in his book 'What is life?' [26]: "The living organism seems to be a macroscopic system which in part of its behavior approaches to that purely mechanical (as contrasted with thermodynamical) conduct to which all systems tend, as the temperature approaches absolute zero and the molecular disorder is removed".

4. The existence of an analogy between the effects of biologically active substances in ultra-low doses or low-intensity physical factors (ionizing radiation and non-ionizing electromagnetic radiation) on biological objects and the features of the interaction between spin structures in superfluid ${ }^{3} \mathrm{He}-\mathrm{B}$ performed through spin supercurrents agrees with the views of some researchers that the physical vacuum has the properties of a superfluid of the ${ }^{3} \mathrm{He}-\mathrm{B}$ type [27-34].

\section{References}

1. Burlakova EB, Konradov AA, Maltseva EL (2008) The effects of ultra-low doses of biologically active substances and low-intensity physical factors. Proceedings of the IV International Symposium on Action Mechanisms of Ultra-low Doses. Russian Academy of Sciences, Moscow: 123-149 (in Russian).

2. Yablokov AV (2002) The Myth of Safety of Low Doses of Radiation. Moscow. (Center for Russian Environmental Policy, plc) "Project-F" (in Russian).

3. Burlakova EB, Konradov AA, Khudyakov IV (1990) Effect of chemical agents in ultralow doses on biological objects. Journal of Nonlinear Biology 1: 77-91.

4. Endler PC, Pongratz W, Smith CW, Schulte J (1995) Non-molecular information transfer from thyroxine to frogs with regard to homeopathic toxicology. Vet Hum Toxicol 37(3): 259-260.

5. Special Reports (1994) Biological effects of low doses of ionizing radiation: A fuller picture. UNSCEAR (United Nations Scientific Committee on the Effects of Atomic Radiation)

6. Mil' EM, Myshlyakova OV, Burlakova EB (2005) Behavior of the p53 Protein under the Action of Weak Physical and Chemical Factors (lonizing Radiation and Antioxidant). Biophysics 50, 1: 69-73.

7. Mil' EM, Albantova AA, Burlakova EB (2008) Ultra-low doses of phenosan cause activation of $\mathrm{p} 53$ protein and bcl-2 with mice $\mathrm{F} 1(\mathrm{CBAxC} 57 \mathrm{BI})$ and AKR. Proceedings of the IV International Symposium on Action Mechanisms of Ultralow Doses. Moscow: the Russian Academy of Sciences: 71-72 (in Russian).

8. Burlakova EB, Goloshchapov AN, Zhizhina GP, Konradov AA (1999) [New aspects of of the effects of low intensity radiation]. Radiats Biol Radioecol 39 26-34

9. Makeev VB, Temur'iants NA (1982) [Frequency dependence of the biological effectiveness of a magnetic field in the range of geomagnetic field micropulsations (0.01-100 Hz)]. Probl Kosm Biol 43: 116-128 (in Russian).

10. Nussbaum RH, Köhnlein W (1994) Inconsistencies and open questions regarding low-dose health effects of ionizing radiation. Environ Health Perspect 102: 656-667.

11. Little JB (1990) Low-dose radiation effects: interactions and synergism. Health Phys 59: 49-55.

12. Ishii K, Hosoi Y, Yamada S, Ono T, Sakamoto K (1996) Decreased incidence of thymic lymphoma in AKR mice as a result of chronic, fractionated low-dose total-body X irradiation. Radiat Res 146: 582-585.

13. Report of the United Nations Scientific Committee on the Effects of Atomic 
Citation: Boldyreva LB (2013) The Physical Aspect of Action of Biologically Active Substances in Ultra-Low Doses and Low-Intensity Physical Factors on Biological Objects: Spin Supercurrents. Altern Integ Med 2: 110. doi:10.4172/2327-5162.1000110

Page 6 of 6

Radiation (2011) Fifty seventh session, includes scientific report "Summary of low-dose radiation effects on health." United Nations, New York.

14. Pagot J (1978) Radiestesie and issuance of form. Maloine, Paris.

15. Korschelt Oskar: Die Nutzbarmachung der lebendigen Kraft des Äthers in der Heilkunst, Landwirtschaft und Technik 2. Auflage ca. 1921 Verlag F.E. Baumann, Bad Schemiedeberg und Leipzig.

16. Grebennikov VS (1984) The secret of breeding sites of solitary bees. Beekeeping 12: 28-29 (in Russian).

17. Imperial Patent Office, Patent No. 69340, issued on 22.06.1893 Class 30: Health Care. Oskar Korschelt in Leipzig. An apparatus for therapeutic purposes without specific or deliberate suggestion. Patented in the German Empire from 14 June 1891 (in German).

18. Grebennicov VS (2006) Flight - Chapter V. (from Grebennicov's book: "My World"). Translated into English from Russian by Dr Cherednichenko. www. keelynet.com/greb/greb.htm. Accessed on March 20, 2012.

19. Borovik-Romanov AS, Bunkov YM, Dmitriev VV, Mukharskiy YM, Sergatskov DA (1989) Investigation of spin supercurrents in ${ }^{3} \mathrm{He}-\mathrm{B}$. Phys Rev Lett 62: 16311634.

20. Dmitriev VV (2005) Spin Superfluidity in 3He. Physics Uspekhi 48: 77-83.

21. Bunkov YM (2009) Spin superfluidity and coherent spin precession. J Phys Condens Matter 21: 164201.

22. Dmitriev VV, Fomin IA (2009) Homogeneously precessing domain in ${ }^{3} \mathrm{He}-\mathrm{B}$ : formation and properties. J Phys Condens Matter 21: 164202.

23. Salomaa MM, Volovik GE (1987) Quantized vortices in superfluid ${ }^{3} \mathrm{He}$. Reviews of Modern Physics 59: 533-604.
24. Mercury intensifies genetic damage caused by radiation (1994, October 24) Sci. and Eng. 23.

25. Bellavite P, Signorine A (2002) Emerging science of homeopathy. North Atlantic Books, Berkeley, California: 6-9.

26. Schrödinger E (1944) What is life? The Physical Aspect of the Living Cell, Cambridge University Press.

27. Sinha KP, Sivaram C, Sudarshan ECG (1976) The Superfluid Vacuum State. Time-Varying Cosmological Constant, and Nonsingular Cosmological Models. Foundations of Physics 6: 717-726.

28. Boldyreva LB, Sotina NB (1992) Superfliud Vacuum with Intrinsic Degrees of Freedom. Physics Essays 5: 510-513.

29. Bauerle C, Bunkov YM, Fisher SN, Godfrin H, Pickett GR (1996) Laboratory simulation of cosmic string formation in the early Universe using superfluid ${ }^{3} \mathrm{He}$. Nature 382: 332

30. Volovic GE (2003) The Universe in a Helium Droplet. Clar Press, Oxford.

31. Winkelmann CB, Elbs J, Bunkov YM, Godfrin H (2006) Probing "cosmological" defects in superfluid ${ }^{3} \mathrm{He}-\mathrm{B}$ with a vibrating-wire resonator. Phys Rev Lett 96 : 205301.

32. Boldyreva LB (2011) An analogy between effects of ultra-low doses of biologically active substances on biological objects and properties of spin supercurrents in superfluid ${ }^{3} \mathrm{He}-\mathrm{B}$. Homeopathy 100: 187-193.

33. Boldyreva LB, Boldyreva EM (2012) The Model of Superfluid Physical Vacuum as a Basis for Explanation of Efficacy of Highly Diluted Homeopathic Remedies. Journal of Homeopathy \& Ayurvedic Medicine 1: 1-6.

34. Boldyreva LB (2012) What does this give to physics: attributing the properties of superfluid ${ }^{3} \mathrm{He}-\mathrm{B}$ to physical vacuum? URSS, Moscow. 